Research Paper

\title{
Upregulation of miRNA3195 and miRNA374b Mediates the Anti-Angiogenic Properties of Melatonin in Hypoxic PC-3 Prostate Cancer Cells
}

\author{
Eun Jung Sohn'1, Gunho Won¹, Jihyun Lee ${ }^{1}$, Sangyoon Lee², Sung-hoon Kim ${ }^{1 凶}$ \\ 1. College of Oriental Medicine, Kyung Hee University, Seoul 130-701, South Korea. \\ 2. Graduate School of East-West Medical Science, Kyung Hee University, Yongin 449-701, Republic of Korea.
}

$\square$ Corresponding author: Sung-Hoon Kim, OMD, Ph.D, E-mail address: sungkim7@khu.ac.kr College of Oriental Medicine, Kyung Hee University, 1 Hoegi-dong, Dongdaemun-gu, Seoul 130-701, Republic of Korea, Tel.: +82-2-961-9233, fax: +82-2-964-1074.

( ) Ivyspring International Publisher. This is an open-access article distributed under the terms of the Creative Commons License (http://creativecommons.org/ licenses/by-nc-nd/3.0/). Reproduction is permitted for personal, noncommercial use, provided that the article is in whole, unmodified, and properly cited.

Received: 2014.05.07; Accepted: 2014.08.13; Published: 2015.01.01

\begin{abstract}
Recently microRNAs (miRNAs) have been attractive targets with their key roles in biological regulation through post-transcription to control mRNA stability and protein translation. Though melatonin was known as an anti-angiogenic agent, the underlying mechanism of melatonin in PC-3 prostate cancer cells under hypoxia still remains unclear. Thus, in the current study, we elucidated the important roles of miRNAs in melatonin-induced anti-angiogenic activity in hypoxic PC-3 cells. miRNA array revealed that 33 miRNAs ( $>2$ folds) including miRNA3195 and miRNA 374b were significantly upregulated and 16 miRNAs were downregulated in melatonin-treated PC-3 cells under hypoxia compared to untreated control. Melatonin significantly attenuated the expression of hypoxia-inducible factor (HIF)-1 alpha, HIF-2 alpha and vascular endothelial growth factor (VEGF) at mRNA level in hypoxic PC-3 cells. Consistently, melatonin enhanced the expression of miRNA3 195 and miRNA 374b in hypoxic PC-3 cells by qRT-PCR analysis. Of note, overexpression of miRNA3195 and miRNA374b mimics attenuated the mRNA levels of angiogenesis related genes such as HIF-1alpha, HIF-2 alpha and VEGF in PC-3 cells under hypoxia. Furthermore, overexpression of miRNA3195 and miRNA374b suppressed typical angiogenic protein VEGF at the protein level and VEGF production induced by melatonin, while antisense oligonucleotides against miRNA 3195 or miRNA 374b did not affect VEGF production induced by melatonin. Also, overexpression of miR3195 or miR374b reduced HIF-1 alpha immunofluorescent expression in hypoxic PC-3 compared to untreated control. Overall, our findings suggest that upregulation of miRNA3195 and miRNA374b mediates anti-angiogenic property induced by melatonin in hypoxic PC-3 cells.
\end{abstract}

Key words: melatonin; miRNA3195; miRNA374b; VEGF; HIF-1 alpha; PC-3 cells.

\section{Introduction}

Melatonin regulates circadian rhythms and modulates various biological functions with anti-cancer (1), anti-aging (2), anti-inflammatory (3), anti-obesity (4) and anti-oxidant $(5,6)$ activities. Regarding the anti-angiogenic activity, melatonin suppresses tumor angiogenesis by inhibiting hypoxia-inducible factor 1 (HIF-1) and vascular endothelial growth factor (VEGF) via sphingosine kinase 1 in HCT116 colon cancer cells (7) and in a mouse tumor model using RENCA renal adenocarcinoma cells (8). In contrast, melatonin promotes osteogenesis during the repair of bone defects (9) and wound healing in an indomethacin-induced gastric ulcer (10) to maintain homeostasis. 
Recently, microRNAs (miRNAs) were shown to be good targets for several intractable diseases, including cancer (11), diabetes (12) and cardiovascular diseases (13). miRNAs, consisting of noncoding small RNAs ( 20-22 nt) and single-stranded RNA molecules, generally regulate gene expression at the post-transcriptional level (14) and play key roles in cell death, hematopoietic lineage differentiation, cell growth, ageing, autophagy $(15,16)$, angiogenesis (17-19) and cancer biology $(20,21)$. miRNAs are transcribed by RNA polymerase II as long primary miRNAs, and can be processed by Drosha, RNAase III endonucleases and DGCR8 (DiGeorge critical region 8 ) in the nucleus (22).

Despite previous reports on 22 miRNAs that are differentially expressed by melatonin treatment in breast cancer cells (23) and EPACs/miRNA-124/Egr1 pathways in neurodegenerative diseases (24), the roles of miRNAs and the anti-angiogenic properties of melatonin remain unclear. In the present study, we examined the profile of differentially expressed miRNAs using a miRNA array in melatonin-treated PC-3 cells and explored the roles of miRNA3195, the most highly upregulated miRNA, and miRNA374b, a predictive biomarker for prostate cancer (25), in the anti-angiogenic activity of melatonin in PC-3 prostate cancer cells using two miRNA overexpression mimics. We demonstrate that melatonin exerts anti-angiogenic activity by upregulating miRNA3195 and miRNA $374 \mathrm{~b}$ in PC-3 prostate cancer cells under hypoxia.

\section{Material and Methods}

\section{Cell culture}

Prostate cancer cell line PC-3 cells were obtained from the American Type Culture Collection (ATCC), and cultured in RPMI1640 medium (Welgene, Daegu, South Korea) supplemented with $10 \%$ fetal bovine serum (FBS), $2 \mu \mathrm{M}$ L-glutamine and penicillin/streptomycin under $5 \% \mathrm{CO}_{2}$ condition.

\section{Hypoxia induction}

For the hypoxic stress, the PC-3 cells in the absence or presence of $1 \mathrm{mM}$ melatonin were cultured for $4 \mathrm{~h}$ under an anaerobic chamber (Forma Scientific, $\mathrm{OH}, \mathrm{USA}$ ) with a humidified atmosphere of $2 \% \mathrm{O}_{2}$ and $5 \% \mathrm{CO}_{2}$ balanced with $\mathrm{N}_{2}$.

\section{miRNA transfection}

The miRNA mimics $(200 \mathrm{nM})$ of miRNA2190, miRNA3195 and miRNA 374b and antisense oligonucleotides $(50 \mathrm{nM})$ against miRNA3195 and miRNA were transfected into PC-3 cells using lipofetamine 2000 (Invitrogen, Carlsbad, CA, USA) reagent according to the manufacture's protocol. Two days or three days after transfection, PC-3 cells were collected for RNA isolation or Protein expression.

\section{Isolation and expression of miRNA}

Total RNA from PC-3 cells in the absence or presence of melatonin (1mM) (Sigma, St. Louis, MO, USA) under hypoxia was isolated by QIAzol (Invitrogen). One microgram of total RNA was used to make cDNA using GenoExplorer TM miRNA cDNA kit (GenoSensor Corporation, Arizona, USA) according to the manufacture's protocol. To determine the expression of miRNAs, qRT-PCR was performed with the LightCycler TM instrument (Roche Applied Sciences, Indianapolis, USA). miRNA primers were purchased from GenoExplorer TM (GenoSensor Corporation, Arizona, USA). U6 or RNU44 was used to normalize the expression of interesting miRNAs.

\section{miRNA microarray analysis}

Total RNA was extracted from the PC-3 cells in the presence or absence of $1 \mathrm{mM}$ melatonin under hypoxia by QIAzol (Invitrogen). For analysis of miRNA expression profile, total RNA sample (100 ng) was labeled with Cyanine 3-pGp (Сy3) using the Agilent miRNA Complete Labeling and Hyb kit (Agilent Technologies, Foster City, CA, USA). The sample was placed on an Agilent Human miRNA v14 (AMDID 026867) and covered by a Gasket slide (Agilent Technologies). Slides were hybridized for $16 \mathrm{~h}$ at $42^{\circ} \mathrm{C}$ in the Agilent hybridization system. Hybridized slides were washed in the first wash buffer $(0.0005 \%$ Triton X-102) for $5 \mathrm{~min}$ and the second wash buffer for $5 \mathrm{~min}$. After the washing steps, the slides were dried by centrifugation at $900 \mathrm{~g}$ at room temperature. Data were obtained as a median of replicated fluorescent signal measurements of the same miRNA from each slide. Slides were analyzed using Exiqon's protocol. Normalization included background subtractions with a global LOWESS regression algorithm. Spots were flagged and analyzed for signal quality (empty spot, spot signal less than background, less than optimal spot morphology, or saturated spot).

\section{Wound healing assay}

Two days after transfection with miRNA3195 and miRNA374b in PC-3 cells, a plastic pipette tip was used to scrape a line between PC-3 cells 2 to $3 \mathrm{~mm}$ wide. The migratory activity of PC-3 cells was evaluated in the miRNA negative control or miRNA mimics. After incubation for $24 \mathrm{~h}$, the PC-3 cells were stained with Diff-Quick solution II (Sysmex CO., Japan). Randomly chosen fields were photographed under an Axiovert S 100 light microscope (Carl Zeiss, Weimar, Germany) at $\times 100$ magnification, and the migrated cells were counted. 


\section{Quantitative real-time PCR assay (qRT-PCR)}

A quantitative real-time PCR was used to detect the expression and the quantification of the targeted molecules such as HIF-1 alpha, HIF-2 alpha and VEGF in $1 \mathrm{mM}$ melatonin-treated PC-3 cells transfected by miRNA3195 and miRNA374b under hypoxia or normoxia. $1 \mu \mathrm{g}$ of total RNA was used to construct the template cDNA with reverse transcription kit (Promega, WI, USA). To amplify the reaction of DNA, RT-qPCR was used with the LightCycler TM instrument (Roche Applied Sciences, Indianapolis, USA) according to the manufacture's protocol. The mRNA level of GAPDH was used to normalize the expression of genes of interest. All reactions were carried out twice, and the final analysis was based on the mean of the reactions. The primers used to be as follows: HIF-1 alpha forward primer 5'-CTGCCACC ACTGATGAATTA-3', reverse primer 5'-GTATGTGG GTAGGAGATGGA-3', HIF-2 alpha forward primer 5'GCGCTAGACTCCGAGAACAT-3', reserve primer 5'-TGGCCACTTACTACCTGACCCTT-3', VEGF forward primer $5^{\prime}$-CCAGCAGAAAGAGGAAAGAG GTAG-3', reverse primer 5'-CCCCAAAAGCAGGTC ACTCAC-3'; GAPDH forward primer: 5'-CCA CTC CTC CAC CTT TGA C-3', reverse primer: 5'-ACC CTG TTG CTG TAG CCA-3'.

\section{Western blotting}

PC-3 cells were exposed to $1 \mathrm{mM}$ melatonin for $24 \mathrm{~h}$ and cell lysates were prepared on ice in lysis buffer containing $50 \mathrm{mM}$ Tris- $\mathrm{HCl}, \mathrm{pH}$ 7.4, $150 \mathrm{mM}$ $\mathrm{NaCl}, 1 \%$ Triton X-100, 0.1\% SDS, 1 mM EDTA, $1 \mathrm{mM}$ $\mathrm{Na}_{3} \mathrm{VO}_{4}, 1 \mathrm{mM} \mathrm{NaF}$, and $1 \mathrm{X}$ protease inhibitor cocktail (Roche, Mannhein, Germany). The lysates were spun at $14,000 \times \mathrm{g}$ for $20 \mathrm{~min}$ at $4^{\circ} \mathrm{C}$ and the supernatants were collected. Protein concentrations were determined by Bradford assay (Bio-Rad, Hercules, CA, USA), and proteins (50-100 $\mu \mathrm{g})$ were separated by electrophoresis on 4-12\% NuPAGE Bis-Tris gels (Novex, Carlsbad, CA, USA). The proteins were then transferred to a Hybond ECL transfer membrane and immunoblotted with antibodies of HIF-1 alpha, VEGF (Santa Cruz Biotechnologies, Santa Cruz, CA, USA), and $\beta$-actin (Sigma, St. Louis, MO, USA).

\section{Measurement of VEGF production by en- zyme-linked immunosorbent assay (ELISA)}

Vascular endothelial growth factor (VEGF) level from the supernatant of PC-3 cells transfected by control, miRNA374b or miRNA3196 mimics or miRNA inhibitors against miRNA374b or miRNA3196 in the presence or absence of melatonin under hypoxia was measured by using VEGF ELISA kit (Invitrogen, Carlsbad, CA, USA) according to the manufacturer's instructions.

\section{Immunofluorescence assay for HIF-1 alpha}

PC-3 cells were exposed to $1 \mathrm{mM}$ melatonin under hypoxia for $4 \mathrm{~h}$ after transfection with miRNA3195 and miRNA374b mimics. PC-3 cells were washed and fixed with 3\% paraformaldehyde and permeabilized in $0.1 \%$ Triton X-100. Incubation with monoclonal anti-HIF1a antibody (Novus Biologicals, clone number H1alpha67) for 30 min was followed by incubation with fluorescein isothiocyanate (FITC)-labeled anti-mouse IgG secondary antibody (Santa Cruz Biotechnology, Santa Cruz, USA). The fixed cells were stained with $5 \mathrm{mg} / \mathrm{ml}$ 4,6-diamidino-2-phenylindole (DAPI; Sigma). Images were photographed under a Delta Vision imaging system (Applied Precision. Issaquah, WA, USA).

\section{Statistical analyses}

Statistical analysis of the data was carried out using Prism (GraphPad software, Inc. CA, USA). All data were presented as means \pm standard error of the mean (SEM). The statistically significant differences between control and melatonin-treated groups were evaluated by the Student's t test and one way Anova Tukey's multiple comparision test.

\section{Results}

Previous studies have shown that melatonin has anti-angiogenic activity by regulating HIF-1alpha and sphingosine kinase 1 pathways $(26,27)$, despite weak cytotoxicity in HCT116 and PC-3 cancer cells. Recently, it was shown that miRNAs play critical roles as modulators of angiogenesis (19). To explore whether melatonin affects angiogenesis by regulating miRNAs, we used a miRNA array to determine miRNA profiles in melatonin-treated PC-3 cells under hypoxia. As shown in Table 1, the levels of 33 miRNAs were significantly upregulated, while those of 16 miRNAs were downregulated in melatonin-treated PC-3 cells based on a miRNA array. The differentially expressed miRNAs were further subjected to gene ontology (GO) and Kyoto Encyclopedia of gene and genomes (KEGG) analysis. Bioinformatic software packages, including TargetScan, miRNAwalk, miRNADB and RNA2-HAS, predicted that the biological processes involved in melatonin-treated PC-3 cells were metabolic process, cellular process, cell communication, developmental process, and transport, whereas the signaling pathways included Wnt, gonadotropin releasing hormone, inflammation mediated by chemokine and cytokine, integrin, cadherin, and angiogenesis-related pathways (Additional file 1: Supplementary Figure 1). Among them, HIF-1 alpha, HIF-2 alpha and VEGF target the angiogenic-signaling pathway. In this study, we found that miR-374b, miR-663 and miR130b are associated with VEGFA; miR-3613-3p 
and miR-3074-3p are associated with HIF-1alpha; hsa-miR-3195_st, miR-21-5p and Let7f are associated with HIF-2alpha; and miR-3613-3p and miR-4710 are associated with HIF-3alpha (Table 2). Among them, we selected two potent microRNAs (miRNA3195 and miRNA374b) for further study based on bioinformatic information and our miRNA data.

We validated upregulation of two miRNAs using qRT-PCR. Consistent with the miRNA array, the levels of miRNA3195 and miRNA374b were upregulated in melatonin-treated PC-3 cells under hypoxia compared to hypoxia control (Figure 1A). We also confirmed that miRNA3195 was upregulated by melatonin in hypoxic DU145 cells compared to normoxic control (Additional file 1: Supplementary Figure 2), while miRNA374b expression was not detected in DU145 cells using RT-qPCR. In addition, miR222 was downregulated in melatonin-treated PC-3 cells under hypoxia, while miR27a was not significantly repressed (Figure 1B).

A
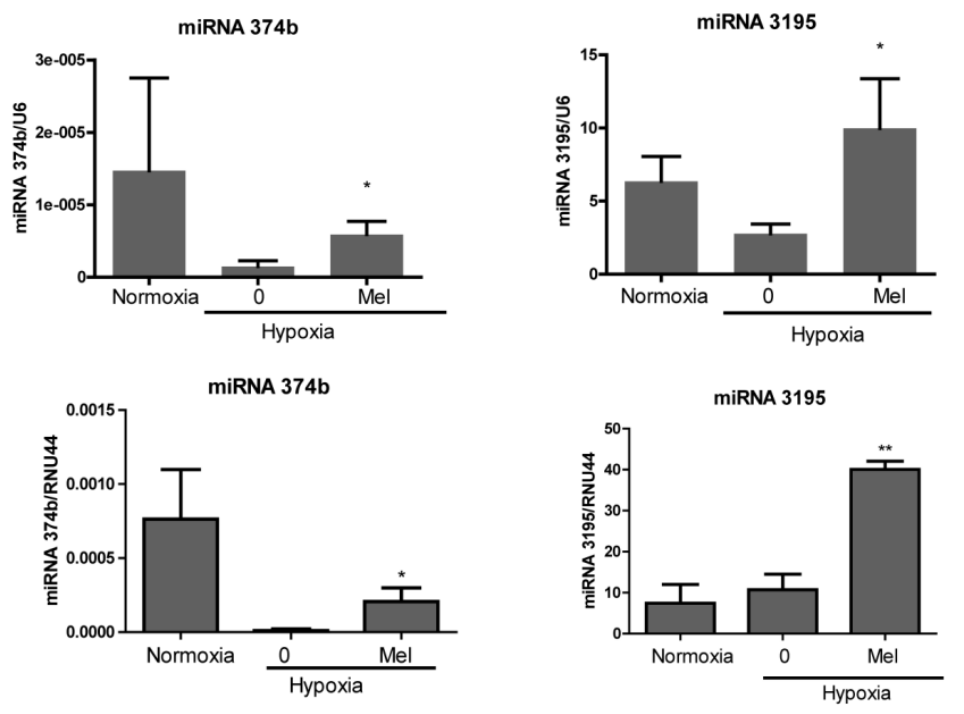

B

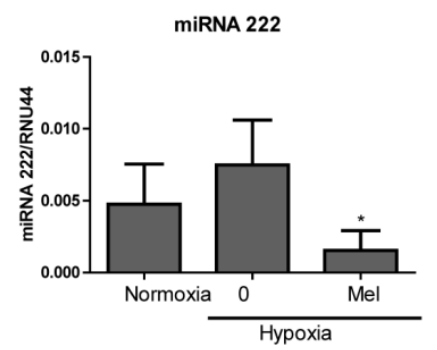

Figure 1. Melatonin upregulated the expression of miRNA3195 and miRNA374b in hypoxic PC-3 cells. PC-3 cells were treated in the absence or presence of melatonin (Mel) $(1 \mathrm{mM}$ ) for $4 \mathrm{~h}$ under hypoxia or normoxia (normal oxygen level condition). Total RNA was isolated and analyzed by qRT-PCR for determining the level of miRNA3195, miRNA374 (A), miRNA222 and miRNA27a (B). U6 or RNU44 was used to normalize the expression of miRNA. Data are presented as means \pm SEM of three independent experiments. ${ }^{*} p<0.05$, ${ }^{* *} p<0.01$ vs hypoxia control. 
Table 1. MicroRNA profile differentially expressed in melatonin treated PC-3 cells under hypoxia.

\begin{tabular}{|lr|lr|}
\hline \multicolumn{1}{|c|}{ Transcript ID } & p > 2 fold & Transcript ID & p $<\mathbf{0 . 5}$ fold \\
\hline hsa-miR-3195_st & 4.252 & hsa-miR-222-star_st & 0.181 \\
hsa-miR-4665-5p_st & 3.711 & hsa-miR-27a-star_st & 0.206 \\
hsa-miR-1246_st & 3.504 & hsa-miR-92a-1-star_st & 0.245 \\
hsa-miR-130b-star_st & 3.317 & hsa-let-7a-2-star_st & 0.309 \\
hsa-miR-4649-5p_st & 3.048 & hsa-miR-3177-3p_st & 0.325 \\
hsa-miR-4492_st & 2.941 & hsa-miR-1226_st & 0.352 \\
hsa-miR-374b_st & 2.674 & hsa-miR-4304_st & 0.356 \\
hsa-miR-4667-5p_st & 2.639 & hsa-miR-23a-star_st & 0.359 \\
hsa-miR-3937_st & 2.618 & hsa-miR-30c-2-star_st & 0.385 \\
hsa-miR-663_st & 2.491 & hsa-miR-1972_st & 0.387 \\
hsa-miR-3074-3p_st & 2.460 & hsa-miR-4436b-5p_st & 0.424 \\
hsa-miR-3175_st & 2.456 & hsa-miR-193a-3p_st & 0.435 \\
hsa-miR-1184_st & 2.394 & hsa-miR-3162-3p_st & 0.445 \\
hsa-miR-3613-3p_st & 2.351 & hsa-miR-1237_st & 0.458 \\
hsa-miR-4488_st & 2.350 & hsa-miR-424-star_st & 0.484 \\
hsa-miR-3621_st & 2.342 & hsa-miR-4671-3p_st & 0.486 \\
hsa-miR-4486_st & 2.340 & & \\
hsa-miR-4710_st & 2.327 & & \\
hsa-miR-192-star_st & 2.320 & & \\
hsa-miR-2116_st & 2.320 & & \\
hsa-miR-1587_st & 2.294 & & \\
hsa-miR-3944-5p_st & 2.284 & & \\
hsa-miR-3196_st & 2.280 & & \\
hsa-miR-4467_st & 2.234 & & \\
hsa-miR-21_st & 2.230 & & \\
hsa-miR-26b_st & 2.218 & & \\
hsa-miR-1973_st & 2.187 & & \\
hsa-miR-4498_st & 2.182 & & \\
hsa-miR-943_st & 2.104 & & \\
hsa-miR-4324_st & 2.104 & & \\
hsa-miR-4750_st & 2.069 & & \\
hsa-miR-3682-3p_st & 2.068 & & \\
hsa-let-7f_st & & & \\
\end{tabular}
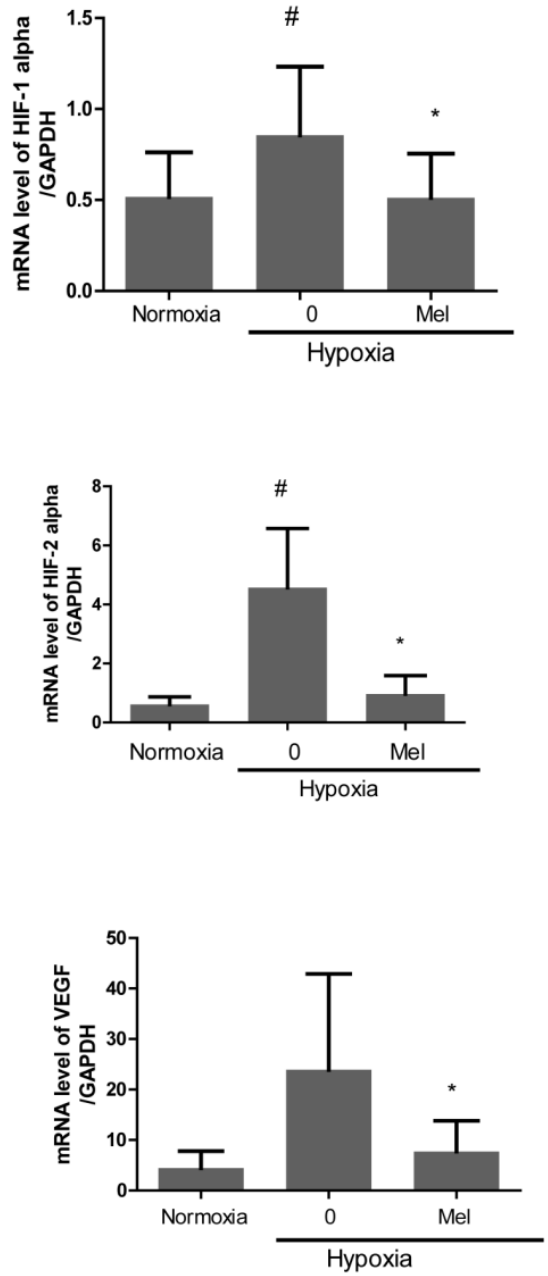

Figure 2. Melatonin attenuated mRNA expression of HIF-1 alpha, HIF-2 alpha and VEGF in hypoxic PC-3. PC-3 cells were treated in the absence or presence of melatonin ( $1 \mathrm{mM}$ ) for $4 \mathrm{~h}$ under hypoxia. Total RNA was analyzed by qRT-PCR to determine the mRNA expression of HIF-1 alpha, HIF-2 alpha and VEGF. GAPDH was used to normalize the target gene. Data are presented as means \pm SEM of three independent experiments. \# $\mathrm{p}<0.05$ vs normoxia control, $* \mathrm{p}<0.05$ vs hypoxia control.

Table 2. Predicted target genes of selected upregulated miRNAs related to angiogenesis.

\begin{tabular}{llll}
\hline & Fold(up) & Predicted molecular target & Source \\
\hline hsa-miR-3195 & 4.251 & HIF2alpha & RNA22-HAS \\
has-miR-374b & 2.674 & VEGFA & miRwalk, Targetscan \\
hsa-miR-663 & 2.674 & VEGFA & miRDB \\
hsa-miR-3613-3p & 2.351 & HIF1alpha & miRDB \\
hsa-miR-4710 & 2.327 & HIF3alpha & miRDB \\
hsa-miR-21-5p & 2.230 & VEGF,HIF1alpha,HIF2alpha & miRwalk \\
hsa-miR-3074-3p & 3.317 & VEGFA & RNA22-HAS \\
hsa-miR-4710 & 3.327 & HIF3alpha & miRDB \\
hsa-miR-1973 & 2.187 & IL-8 & miRDB \\
has-miR-943 & 2.104 & HIF3alpha & RNA22-HAS \\
Let7f & 2.055 & HIF2alpha,HIF3alpha & RNA22-HSA \\
\hline
\end{tabular}


A
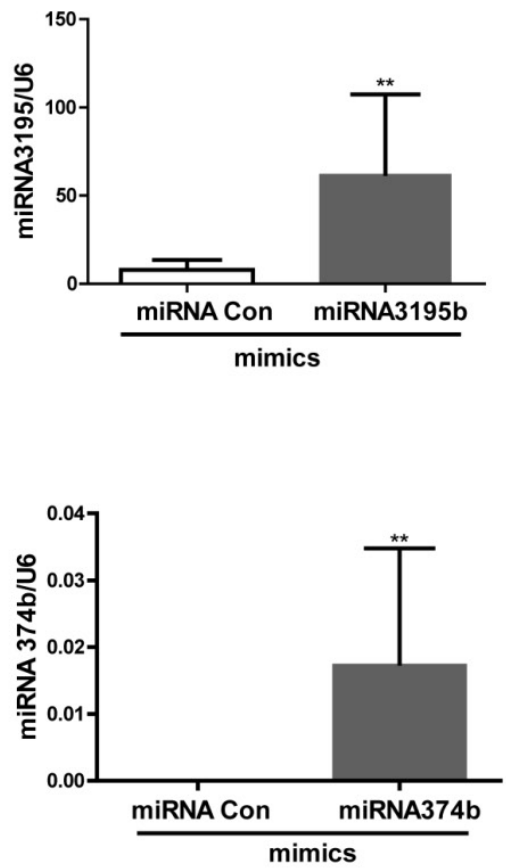

C

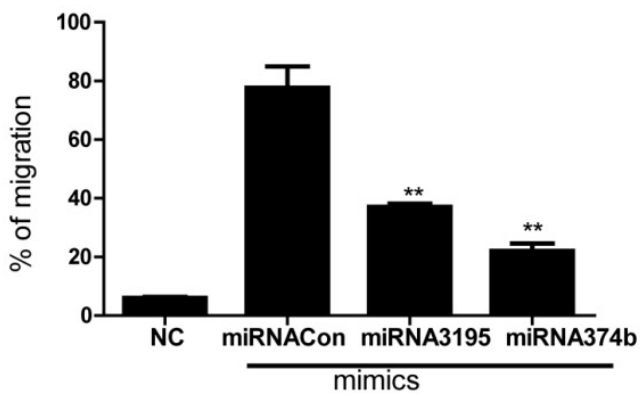

B
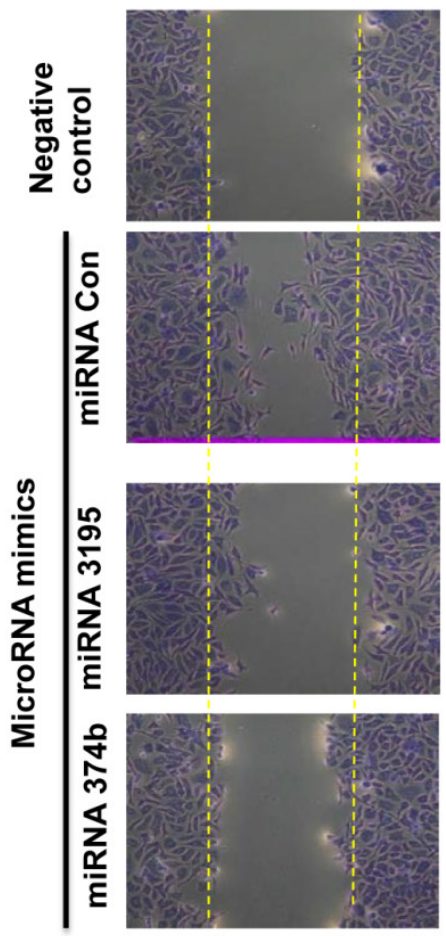

D

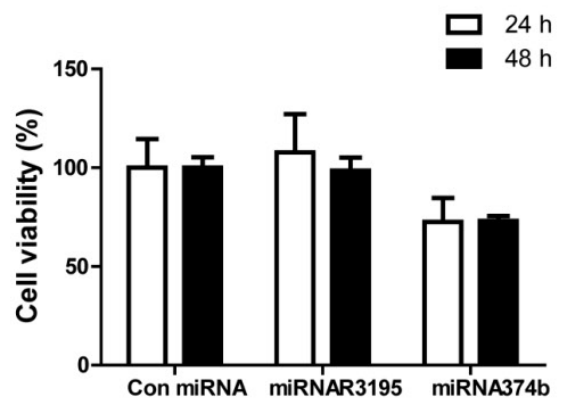

Figure 3. Overexpression of miRNA3195 and miRNA374b suppressed the migratory activity of hypoxic PC-3 cells by wound healing assay. Mimics of miRNA3195 and miRNA374b were transfected into PC-3 cells. Two days after transfection, the cells were harvested. (A) Overexpression level of miRNA3195 and miRNA374b after transfection with miRNA3195 and miRNA374b mimics. Total RNAs for miRNA3195 and miRNA374b were analyzed by qRT-PCR. (B) Effect of overexpressed miRNA3195 and miRNA374b on migratory activity of PC-3 cells under normoxia condition. Two days after transfection with miRNA mimics, the confluent PC-3 cells were scratched with a tip and the migratory activity of PC-3 cells was evaluated under an inverted microscopy. Negative control (NC) represents non-migratory status right after wound scratch in the PC-3 cells. (C) Bar graphs showed quantification of migrated PC-3 cells. (D)Cell viability in miRNA3195 and miRNA374b transfected PC-3cells. After transfection with control vector, miRNA3195, or miRNA374b mimics, MTT assay was performed at 24 or $48 \mathrm{~h}$ after transfection. Data are presented as means \pm SEM of triplicate samples. **, $\mathrm{P}$ $<0.01$ vs. miRNA control

Likewise, overexpression of miRNA3195 and miRNA374b effectively inhibited mRNA expression of HIF-1alpha, HIF-2alpha and VEGF in hypoxic PC-3 cells compared to untreated hypoxia control (Figure 4A, 4B, 4C). In addition, Western blotting revealed that expression levels of HIF-1alpha and VEGF were attenuated in miRNA3195 and miRNA374b mimic-transfected PC-3 cells under hypoxia, but not in miRNA1290-transfected PC-3 cells (Figure 4D). Of note, melatonin significantly reduced VEGF produc- tion (as determined by ELISA) and VEGF protein (as determined by Western blotting) in hypoxic PC-3 cells transfected with miRNA3195 and miRNA374b mimics compared to miRNA vector control or only melatonin treated control (Figure 5A and C), while antisense oligonucleoties against miRNA374b or miRNA3195 did not attenuate production of VEGF in the presence of melatonin (Figure 5B). Antisense oligonucleoties against miRNA374b without melatonin rather increased the production of VEGF (Figure 5B). 
In contrast, the miRNA1290 mimic (used as the negative control) did not affect VEGF protein expression (Figure 5C). Similarly, immunofluorescence assay revealed that melatonin attenuated HIF-1alpha expression in miRNA3195 and miRNA 374b mimic-transfected PC-3 cells under hypoxia, whereas the miRNA1290 mimic did not affect VEGF immunofluorescent expression (Figure 5D).

\section{Discussion}

Many studies have demonstrated a dual effect of melatonin on angiogenesis in cancer and endothelial cells. Ganguly et al. (10) reported that melatonin promotes angiogenesis during protection and healing of indomethacin-induced gastric ulcers, and Soybir et al. (28) suggested that melatonin enhances neovascularization and wound healing in rats with $5-\mathrm{cm}$ incision damage. In contrast, melatonin suppressed tumor angiogenesis in HepG2 hepatocellular cancer (29) and PANC-1 pancreatic cancer (30) by inhibiting VEGF or signal transducer and activator of transcription 3 (STAT3) and HIF-1 alpha, and in PC-3 prostate cancer by inhibiting the sphingosine kinase pathway

A

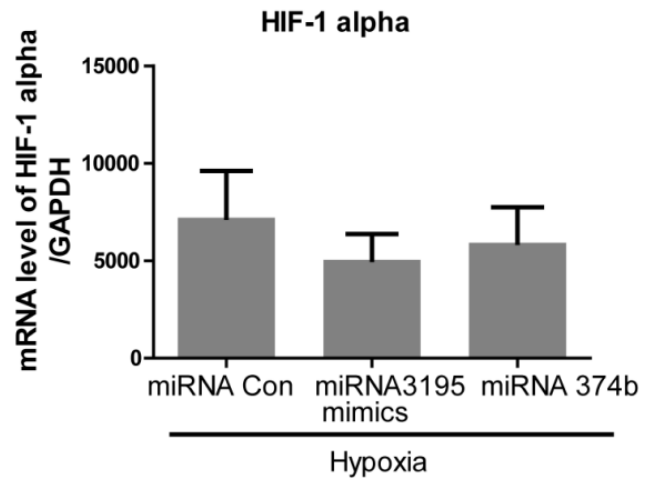

C

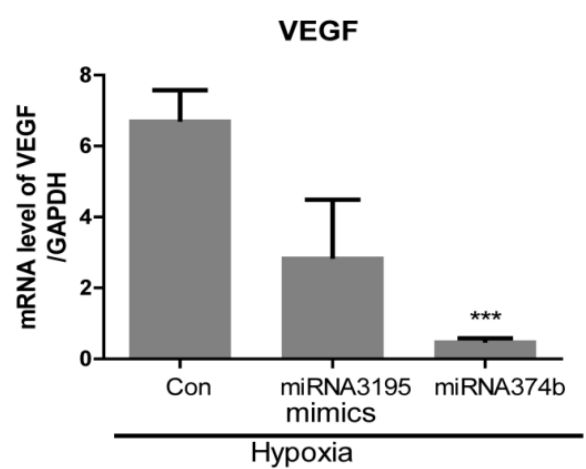

(26). Nevertheless, the mechanism underlying the association between melatonin and miRNAs remains unknown. In the current study, we found that 33 miRNAs (changes of at least twofold), including miRNA3195 and miRNA 374b, were significantly upregulated, and 16 miRNAs were downregulated, in melatonin-treated PC-3 cells under hypoxia based on miRNA expression profiling using microarrays, implying the important roles of miRNAs in melatonin-treated PC-3 cells under hypoxia. Considering our previous report that melatonin suppresses HIF-1alpha accumulation by inhibiting the sphingosine kinase 1 (SPHK1) pathway and reactive oxygen species (ROS) generation in hypoxic PC-3 cells, the anti-angiogenic activity may be the result of blockade of HIF-1alpha/VEGF/SPHK1 axis in association with some key miRNAs in hypoxic PC-3 cells. We selected two potent miRNAs (miRNA3195 and miRNA374b) for further mechanistic studies since miRNA3195 was the most highly upregulated based on our microRNA array data analysis and miRNA374b is downregulated in human prostate cancer tissues (25).

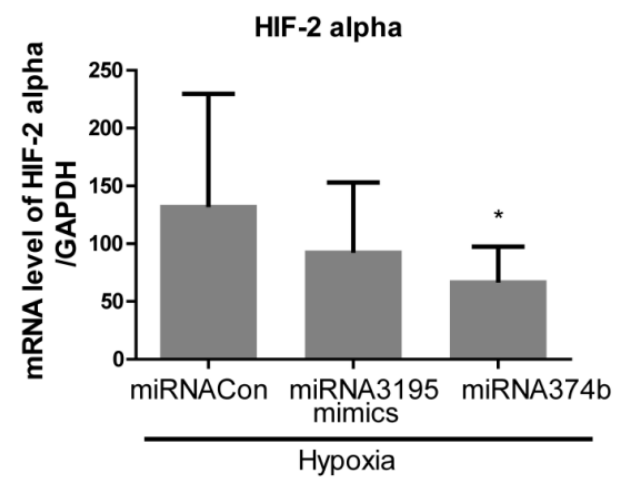

D

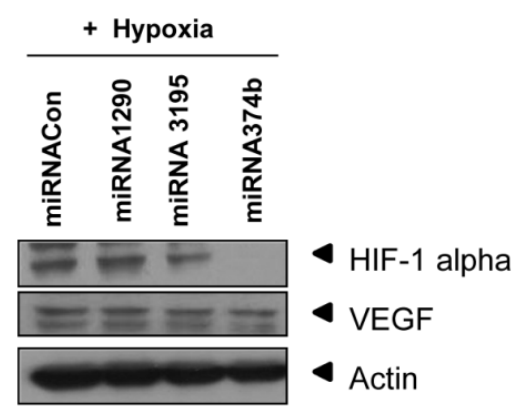

Figure 4. Overexpression of miRNA3195 and miRNA374b inhibited the expression of HIF-1 alpha, HIF-2 alpha and VEGF in hypoxic PC-3 cells. Effect of miRNA3195 and miRNA374b mimics on HIF-1 alpha (A), HIF-2 alpha (B) and VEGF (C) in hypoxic PC-3 cells by qRT-PCR. Mimics of miRNA3195 and miRNA374b were transfected into PC-3 cells. Two days after transfection, PC-3 cells were cultured for $4 \mathrm{~h}$ under hypoxia. Total RNAs were isolated and analyzed to determine the mRNA expression of HIF-lalpah, HIF-2alpha and VEGF by qRT-PCR. GAPDH was used to normalize the target gene. Data are presented as means \pm SEM of three independent experiments. (D) Effect of miRNA2190 (negative control), miRNA3195 and miRNA374b mimics on HIF1 alpha and VEGF in hypoxic PC-3 cells by Western blotting. 
A

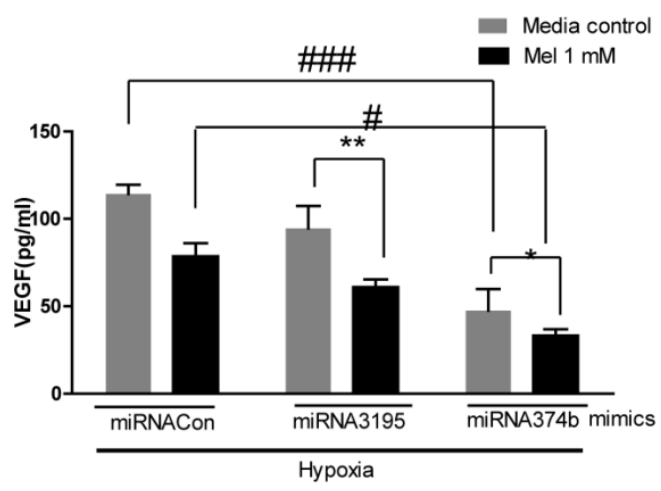

C

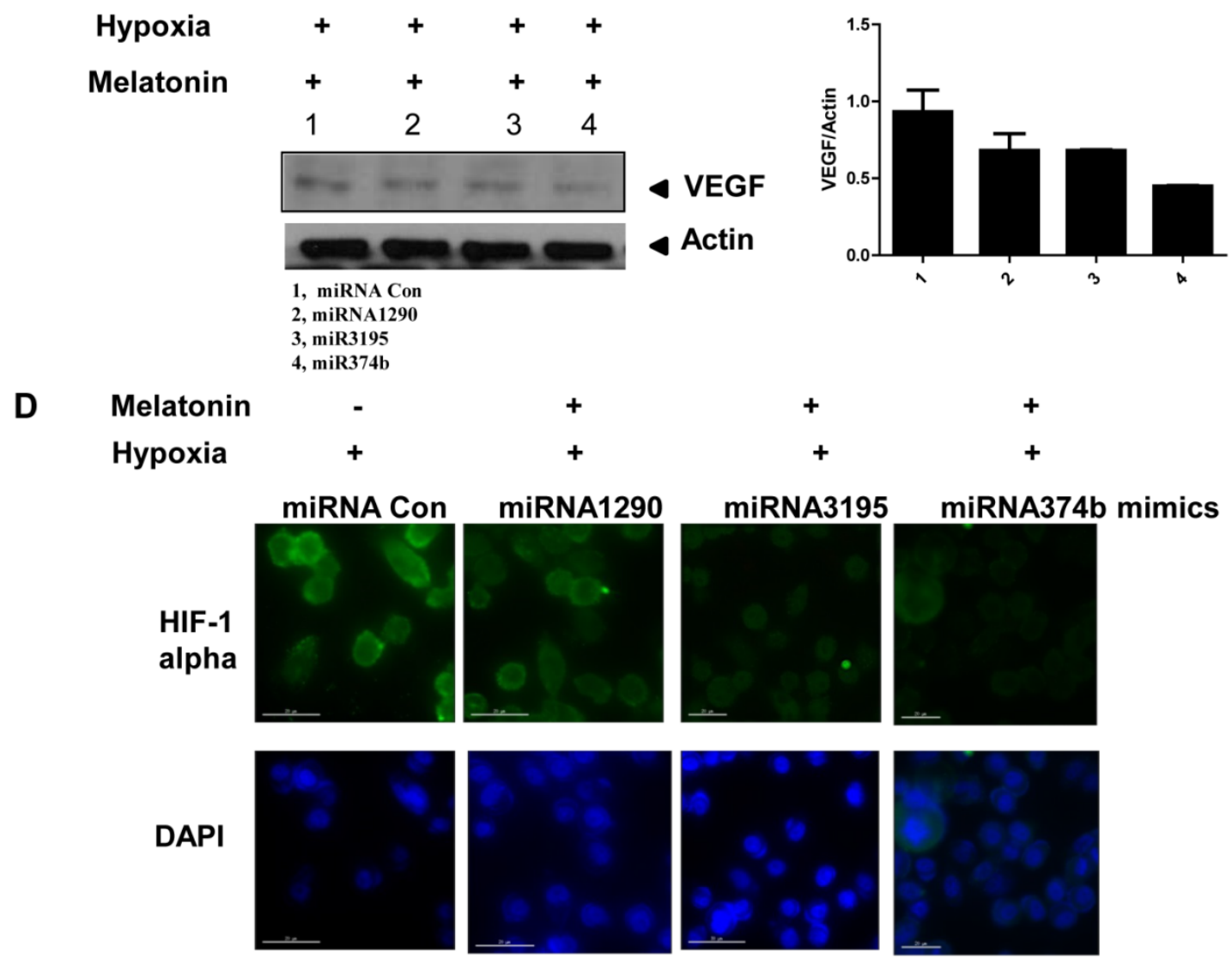

Figure 5. Melatonin significantly reduced VEGF secretion and HIF-1 alpha green color expression in miRNA3195 and miRNA374b mimics treated in hypoxic PC-3 cells. Effect of melatonin on VEGF production in hypoxic PC-3 cells transfected with miR3195 or miRNA374b mimics(A) or anti-senese olionucelotides aganist miRNA3195 or miRNA374b(B). Mimics or anti-senese olionucelotides of miRNA3195 and miRNA374b were transfected into PC-3 cells. Two days after transfection, the cells were cultured in the presence of melatonin at $1 \mathrm{mM}$ for $4 \mathrm{~h}$ under hypoxia. The level of VEGF was measured by using VEGF ELISA kits. Statistical significance was evlauted using Tukey's multiple comparison test: \# $p<0.05$, $p<0.001$ vs. hypoxic control, and ${ }^{*} \mathrm{p}<0.05$, * $\mathrm{p}<0.01$ vs. microRNA control. Data are presented as mean \pm SEM for triplicate experiment. (C) Effect of melatonin on VEGF production assessed by Western blotting in hypoxic PC-3 cells when miRNA3195 and miRNA374b were overexpressed using mimics. PC-3 cells were exposed to $1 \mathrm{mM}$ melatonin for $24 \mathrm{~h}$ and Western blotting was performed as shown in Materials and methods. 1 st lane; hypoxia, $2^{\text {nd }}$ lane; hypoxia + miRNA1 290 mimic as negative control. 3rd lane; hypoxia + miRNA3195 mimic, 4th lane; hypoxia + miRNA374b. (D) Effect of melatonin on HIF-1 alpha by immunofluorescent green color expression in miRNA3195 and miRNA374b mimics treated in hypoxic PC-3 cells by immunofluorescence assay. 


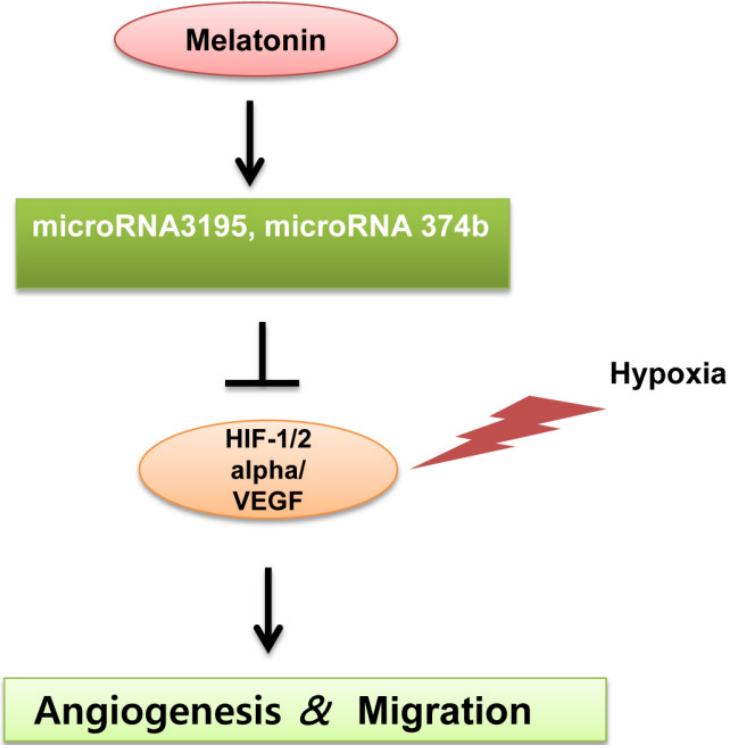

Figure 6. A schematic diagram for anti-angiogenic property of melatonin via regulation of miRNA3195 and miRNA374b in hypoxic PC-3 cells.

Consistent with the miRNA array data, qRT-PCR analysis demonstrated that melatonin enhanced the expression of miRNA3195 and miRNA374b. In addition, wound-healing assays revealed that melatonin significantly suppressed the repair motility of hypoxic PC-3 cells, indicating that melatonin has antimigratoty activity, since angiogenic capillary sprouts invade the fibrin/fibronectin-rich clot during wound healing and within a few days to organize into a microvascular network throughout the granulation tissue (31-33). HIF-1alpha promotes the expression of pro-angiogenic genes, such as VEGFA, PDGFB and IL-8 $(34,35)$, and both HIF-1 alpha and HIF-2 alpha regulate the expression of genes involved in angiogenesis (36). Thus, qRT-PCR analysis revealed that melatonin significantly attenuated the expression of HIF-1alpha, HIF-2alpha and VEGF at the mRNA level in hypoxic PC-3 cells, which was supported by previous reports that melatonin exerts an anti-angiogenic effect by decreasing VEGF or HIF-1alpha expression under hypoxic conditions in vitro (7) and in vivo (37).

Previous studies demonstrate that miRNAs play important roles in angiogenesis, which involves the formation of new blood vessels from pre-existing blood vessels $(38,39)$. Furthermore, several studies have shown that miRNAs are upregulated or downregulated in hypoxic breast and colon cancer cells (40). In addition, the hypoxia-regulated miRNAs miRNA-15b, miRNA-16b, and miRNA20a/b are known to control VEGF production, because VEGF (a pro-angiogenic molecule) has been identified as a potential miRNA target (41). Nevertheless, no report of the relationship between melatonin and miRNAs has been published to date except one report on the regulation of melatonin synthesis by miR-483 (42). In the present study, overexpression mimics of miRNA3195 and miRNA374b in PC-3 cells abrogated the mRNA levels of the angiogenesis-related proteins HIF-1alpha, HIF-2alpha and VEGF under hypoxia, demonstrating that miRNA3195 and miRNA374b target the HIF-1/2 alpha/VEGF axis. Consistently, melatonin attenuated VEGF expression at the protein level, reduced VEGF secretion, and also reduced HIF-1 alpha immunofluorescent expression in hypoxic PC-3 cells transfected with miRNA3195 and miRNA374b mimics compared to untreated control, indicating the antiangiogenic property of miRNA3195 and miRNA374b. Also, we confirmed that miRNA3195 was upregulated by melatonin in hypoxic DU145 cells. Furthermore, melatonin upregulated miRNA3195 and miRNA374b as determined by miRNA microarray and qRT-PCR analysis and overexpression of miRNA3195 and miRNA374b synergistically reduced VEGF production by hypoxic PC-3 prostate cancer cells, implying important role of miRNA3195 and miRNA374b in melatonin induced antiangiogenic activity.

In summary, melatonin significantly attenuated the expression of HIF-1 alpha, HIF-2 alpha and VEGF at mRNA level and also enhanced the expression of miRNA3195 and miRNA 374b in hypoxic PC-3 cells by qRT-PCR analysis. Notably, overexpression of miRNA3195 and miRNA374b mimics attenuated the mRNA levels of HIF-1alpha, HIF-2 alpha and VEGF in hypoxic PC-3 cells. Furthermore, overexpression of miRNA3195 and miRNA374b suppressed VEGF protein expression and production and also reduced HIF-1 alpha immunofluorescent expression in melatonin treated PC-3 cells under hypoxia. Taken togetherl, our findings demonstrate that upregulation of miRNA3195 and miRNA374b mediates anti-angiogenic property induced by melatonin in hypoxic PC-3 cells. However, further mechanistic studies are required to confirm the anti-angiogenic effects of miRNA3195 and miRNA374b in animals.

\section{Supplementary Material}

Additional File 1:

Supplementary Figures 1-2.

http://www.jcancer.org/v06p0019s1.pdf

\section{Acknowledgements}

This work was supported by the National Research Foundation of Korea (NRF) grant funded by the Korea government (MSIP) (No. No. 2012-0005755).

\section{Conflict of Interest}

The authors have declared that there is no conflict of interest. 


\section{References}

1. Sanchez-Hidalgo M, Guerrero JM, Villegas I, Packham G, de la Lastra CA. Melatonin, a natural programmed cell death inducer in cancer. Curr Med Chem 2012;19(22):3805-3821.

2. Pandi-Perumal SR, BaHammam AS, Brown GM, Spence DW, Bharti VK, Kaur $C$, et al. Melatonin antioxidative defense: therapeutical implications for aging and neurodegenerative processes. Neurotox Res 2013;23(3):267-300.

3. Carrillo-Vico A, Lardone PJ, Alvarez-Sanchez N, Rodriguez-Rodriguez A, Guerrero JM. Melatonin: buffering the immune system. Int J Mol Sci 2013;14(4):8638-8683.

4. Rastmanesh R, de Bruin PF. Potential of melatonin for the treatment or prevention of obesity: an urgent need to include weight reduction as a secondary outcome in clinical trials of melatonin in obese patients with sleep disorders. Contemp Clin Trials 2012;33(4):574-575.

5. Galano A, Tan DX, Reiter RJ. On the free radical scavenging activities of melatonin's metabolites, AFMK and AMK. J Pineal Res 2013;54(3):245-257.

6. Galano A, Tan DX, Reiter RJ. Melatonin as a natural ally against oxidative stress: a physicochemical examination. J Pineal Res 2012;51(1):1-16.

7. Park SY, Jang WJ, Yi EY, Jang JY, Jung Y, Jeong JW, et al. Melatonin suppresses tumor angiogenesis by inhibiting HIF-1alpha stabilization under hypoxia. $J$ Pineal Res 2010;48(2):178-184

8. Kim KJ, Choi JS, Kang I, Kim KW, Jeong CH, Jeong JW. Melatonin suppresses tumor progression by reducing angiogenesis stimulated by HIF-1 in a mouse tumor model. J Pineal Res 2013;54(3):264-270.

9. Ramirez-Fernandez MP, Calvo-Guirado JL, de-Val JE, Delgado-Ruiz RA, Negri B, Pardo-Zamora G, et al. Melatonin promotes angiogenesis during repair of bone defects: a radiological and histomorphometric study in rabbit tibiae. Clin Oral Investig 2012;17(1):147-158.

10. Ganguly K, Sharma AV, Reiter RJ, Swarnakar S. Melatonin promotes angiogenesis during protection and healing of indomethacin-induced gastric ulcer: role of matrix metaloproteinase-2. J Pineal Res 2010;49(2):130-140.

11. An J, Zhu X, Wang H, Jin X. A dynamic interplay between alternative polyadenylation and microRNA regulation: implications for cancer (Review). Int $J$ Oncol 2013;43(4):995-1001.

12. Guay C, Roggli E, Nesca V, Jacovetti C, Regazzi R. Diabetes mellitus, a microRNA-related disease? Transl Res 2011;157(4):253-264.

13. Dominguez-Rodriguez A. Melatonin in cardiovascular disease. Expert Opin Investig Drugs 2012;21(11):1593-1596.

14. Bartel DP. MicroRNAs: genomics, biogenesis, mechanism, and function. Cell 2004;116(2):281-297.

15. Schickel R, Boyerinas B, Park SM, Peter ME. MicroRNAs: key players in the immune system, differentiation, tumorigenesis and cell death. Oncogene 2008;27(45):5959-5974.

16. Croce CM, Calin GA. miRNAs, cancer, and stem cell division. Cell 2005;122(1):6-7.

17. Finn NA, Searles CD. Intracellular and Extracellular miRNAs in Regulation of Angiogenesis Signaling. Curr Angiogenes 2012;4(102):299-307.

18. Kuehbacher A, Urbich C, Zeiher AM, Dimmeler S. Role of Dicer and Drosha for endothelial microRNA expression and angiogenesis. Circ Res 2007;101(1):59-68.

19. Landskroner-Eiger S, Moneke I, Sessa WC. miRNAs as modulators of angiogenesis. Cold Spring Harb Perspect Med 2013;3(2):a006643.

20. Hammond SM. MicroRNAs as tumor suppressors. Nat Genet 2007;39(5):582-583

21. Zhang B, Pan X, Cobb GP, Anderson TA. microRNAs as oncogenes and tumor suppressors. Dev Biol 2007;302(1):1-12.

22. Gregory RI, Yan KP, Amuthan G, Chendrimada T, Doratotaj B, Cooch N, et al. The Microprocessor complex mediates the genesis of microRNAs. Nature 2004;432(7014):235-240.

23. Lee SE, Kim SJ, Youn JP, Hwang SY, Park CS, Park YS. MicroRNA and gene expression analysis of melatonin-exposed human breast cancer cell lines indicating involvement of the anticancer effect. J Pineal Res 2011;51(3):345-352.

24. Wang X, Wang ZH, Wu YY, Tang H, Tan L, Gao XY, et al. Melatonin attenuates scopolamine-induced memory/synaptic disorder by rescuing EPACs/miR-124/Egr1 pathway. Mol Neurobiol 2013;47(1):373-381.

25. He HC, Han ZD, Dai QS, Ling XH, Fu X, Lin ZY, et al. Global analysis of the differentially expressed miRNAs of prostate cancer in Chinese patients. BMC Genomics 2013;14:757.

26. Cho SY, Lee HJ, Jeong SJ, Kim HS, Chen CY, Lee EO, et al. Sphingosine kinase 1 pathway is involved in melatonin-induced HIF-1alpha inactivation in hypoxic PC-3 prostate cancer cells. J Pineal Res 2011;51(1):87-93.

27. Zhang Y, Liu Q, Wang F, Ling EA, Liu S, Wang L, et al. Melatonin antagonizes hypoxia-mediated glioblastoma cell migration and invasion via inhibition of HIF-1alpha. I Pineal Res 2013

28. Soybir G, Topuzlu C, Odabas O, Dolay K, Bilir A, Koksoy F. The effects of melatonin on angiogenesis and wound healing. Surg Today 2003;33(12):896-901.

29. Carbajo-Pescador S, Ordonez R, Benet M, Jover R, Garcia-Palomo A, Mauriz JL, et al. Inhibition of VEGF expression through blockade of Hif1alpha and STAT3 signalling mediates the anti-angiogenic effect of melatonin in HepG2 liver cancer cells. Br J Cancer 2013;109(1):83-91.

30. Cui P, Yu M, Peng X, Dong L, Yang Z. Melatonin prevents human pancreatic carcinoma cell PANC-1-induced human umbilical vein endothelial cell pro- liferation and migration by inhibiting vascular endothelial growth factor expression. J Pineal Res 2012;52(2):236-243.

31. Qiu JG, Factor S, Chang TH, Knighton D, Nadel H, Levenson SM. Wound healing: captopril, an angiogenesis inhibitor, and Staphylococcus aureus peptidoglycan. J Surg Res 2000;92(2):177-185.

32. Tonnesen MG, Feng X, Clark RA. Angiogenesis in wound healing. J Investig Dermatol Symp Proc 2000;5(1):40-46.

33. Bloch W, Huggel K, Sasaki T, Grose R, Bugnon P, Addicks K, et al. The angiogenesis inhibitor endostatin impairs blood vessel maturation during wound healing. FASEB J 2000;14(15):2373-2376.

34. Hellwig-Burgel T, Stiehl DP, Wagner AE, Metzen E, Jelkmann W. Review: hypoxia-inducible factor-1 (HIF-1): a novel transcription factor in immune reactions. J Interferon Cytokine Res 2005;25(6):297-310.

35. Weijts BG, Bakker WJ, Cornelissen PW, Liang $\mathrm{KH}$, Schaftenaar $\mathrm{FH}$, Westendorp B, et al. E2F7 and E2F8 promote angiogenesis through transcriptional activation of VEGFA in cooperation with HIF1. EMBO J 2012;31(19):3871-3884.

36. Giatromanolaki A, Koukourakis MI, Sivridis E, Turley H, Talks K, Pezzella F, et al. Relation of hypoxia inducible factor 1 alpha and 2 alpha in operable non-small cell lung cancer to angiogenic/molecular profile of tumours and survival. Br J Cancer 2001;85(6):881-890.

37. Kaur C, Sivakumar V, Lu J, Ling EA. Increased vascular permeability and nitric oxide production in response to hypoxia in the pineal gland. J Pineal Res 2007;42(4):338-349.

38. Hong L, Han Y, Zhou Y, Nita A. Angiogenesis-related microRNAs in colon cancer. Expert Opin Biol Ther 2013;13(1):77-84.

39. Madanecki P, Kapoor N, Bebok Z, Ochocka R, Collawn JF, Bartoszewski R. Regulation of angiogenesis by hypoxia: the role of microRNA. Cell Mol Biol Lett 2013;18(1):47-57.

40. Kulshreshtha R, Davuluri RV, Calin GA, Ivan M. A microRNA component of the hypoxic response. Cell Death Differ 2008;15(4):667-671.

41. Hua Z, Lv Q, Ye W, Wong CK, Cai G, Gu D, et al. MiRNA-directed regulation of VEGF and other angiogenic factors under hypoxia. PLoS One 2006;1:e116.

42. Clokie SJ, Lau P, Kim HH, Coon SL, Klein DC. MicroRNAs in the pineal gland: miR-483 regulates melatonin synthesis by targeting arylalkylamine N-acetyltransferase. J Biol Chem 2012;287(30):25312-25324. 